\title{
$\mathbf{C P} \# \overline{\mathbf{C P}^{2}}$ 中的光滑 2- 纽结
}

\author{
高红铸
}

(北京师范大学数学系, 北京 100875)

\section{关键词 2-纽结、球丛、同痕类、四维流形、同肧}

本文对 $\mathbb{C} P^{2} \# \overline{\mathbb{C} P^{2}}$ 中具有单连通补的 2- 纽结给出了一个完整的分类. 首先简单回顾了 $S^{2}$ 上的球丛的知识, 并解释了我们. 以后要用的符号, 然后是主要引理和定理的叙述及证明.

\section{$1 S^{2}$ 上的 球 丛}

在这一节中, 我们对 $S^{2}$ 上的 $S^{2}$ - 球丛做一个简单回顾. 详细内容参见文献[1] 和 [2]. $S^{2}$ 上 的 $S O(3)$ 丛的等价类 1-1 对应于 $\pi_{1}(S O(3))=Z_{2}$. 因此 $S^{2}$ 上只有唯一的非平凡 $S^{2}$ - 球丛, 记为 $T$. 记 $P$ 为平凡球丛 $S^{2} \times S^{2}$. 因为 $\pi_{1}(S O(2))=Z$, 故对应于每个整数, 都有一个 $S^{2}$ 上的 $S O(2)-$ 丛. 注意满射 $\pi_{1}(S O(2)) \rightarrow \pi_{1}(S O(3))$ 意味着 $P$ 和 $T$ 均可约化为 $S O(2)$ - 丛. 将 $S O(2)$ 视为 $S O(3)$ 中的圆. 设 $T_{k}$ 为度数是 $k$ 的标准映射, 它将 $S^{2}$ 的大圆映上 $S O(2)$. 记 $B_{k}$ 为 $S^{2}$ 上以 $S^{2}$ 为纤 维, $S O(2)$ 为作用群, $T_{k}$ 为示性映射的丛. 因为 $T_{k}$ 代表元素 $k \in \pi_{1}(S O(2))=Z$, 所以 $B_{k}$ 根据 $k$ 是 偶的或奇的分别对应着 $P$ 或 $T . S O(2)$ 在 $S^{2}$ 上的作用保持南极和北极 $S^{0} \subset S^{2}$ 不动. 因而决定 了 $B_{k}$ 的一个子丛, 它是平凡丛 $S^{2} \times S^{0}$. 从而 $B_{k}$ 有截面. 记 $\sum_{\mathrm{k}}$ 为对应着 “南”极的截面. 则 $\sum_{k}$ 是一个嵌人球, 它代表 $H_{2}\left(B_{k}, Z\right)$ 中的一个类. 若记 $\sigma_{k}$ 为对应于纤维的嵌人球, 那么 $\sum_{k}$ 和 $\sigma_{k}$ 生 成 $H_{2}\left(B_{k}, Z\right)$, 并有相交数的关系: $\sigma_{k} \cdot \sigma_{k}=0, \sigma_{k} \cdot \sum_{k}=1$ 及 $\sum_{k} \cdot \sum_{k}=$ 相应的 $S O(2)$ - 丛有截 面的阻碍, 即 Euler 数 $=k . P$ 和 $T$ 既可约化为 $S O(2)$ 丛 $B_{0}$ 和 $B_{1}$, 又可分别约化为 $B_{2 k}$ 和 $B_{2 k+1}$. 其第二同调群的基 $\sigma_{i}$ 和 $\sum_{i}$ 有如下关系: $\sum_{2 k}=\sum_{0}+k \sigma_{0}, \sigma_{2 k}=\sigma_{0} ; \sum_{2 k+1}=\sum_{1}+k \sigma_{1}, \sigma_{2 k+1}=\sigma_{1}$.

设 $C P^{2}$ 为具有自然定向的复投影平面, $\overline{\mathrm{C} P^{2}}$ 具有相反的定向. 在 $B_{1}$ 中, $S O(2)$ 在纤维 $S^{2}$ 上 的作用将正交于 $S^{0}$ 的大圆 $S^{1} \subset S^{2}$ 作用到它本身. 从而决定了 $B_{1}$ 的一个子丛. 它是 $S^{2}$ 上的 $S^{1}$ 丛, 有 Euler 数 1. 从而是 Hopf 丛 $S^{3} \rightarrow S^{2}$. 大圆 $S^{1}$ 将 $B_{1}^{1}$ 的纤维分为上、下两个半球, $S O(2)$ 将每一个作用到自身, 从而决定了两个等价的子丛 $B_{1}^{+}$和 $B_{1}^{-}$. 它们是 $S^{2}$ 上的 $D^{2}$ - 丛, 以 $S^{3}$ 作 为公共边界, 因而都微分同胚于 $\mathbf{C} P^{1}$ 在 $\mathbf{C} P^{2}$ 中的一个管状邻域. 注意到定向后, 即有它微分同 胚于 $\mathbf{C} P^{2} \# \overline{\mathbf{C} \boldsymbol{P}^{2}}$. 如果记 $\xi, \eta$ 为 $H_{2}\left(\mathbf{C} P^{2} \# \overline{\mathbf{C} P^{2}}, Z\right)$ 的自然生成元, 它们分别表示 $\mathbf{C} P^{2}$ 和 $\overline{\mathbf{C} P^{2}}$ 中的 复直线, 则在以上的微分同胚中, $\xi$ 映到 $\sum_{1}$ 而 $\eta$ 映到 $\sigma_{1}-\sum_{1}$.

\section{$2 \mathrm{C} P^{2} \# \overline{\mathrm{C} P^{2}}$ 中的光滑 2- 纽结}

设 $\xi, \eta$ 是 $H_{2}\left(\mathbf{C} P^{2} \# \overline{\mathbf{C} P^{2}}, z\right)$ 的自生成元, $\xi \cdot \xi=-\eta \cdot \eta=1, \xi \cdot \eta=0 . u=p \xi+q \eta$ 可表示

1993-05-14 收稿. 
为嵌人球当且仅当 ||$p|-| q|| \leqslant 1$ 或 $(p, q)=( \pm 2,0)$ 或 $(0, \pm 2)$ (见文献[3]). 设 $S$ 是 $\mathbf{C} P^{2} \# \overline{\mathbf{C} P^{2}}$ 中 的光滑嵌人球, 它表示同调类 $u \in H_{2}\left(\mathbf{C} P^{2} \# \overline{\mathbf{C} P^{2}}, Z\right)$. 则我们称 $S=\left(\mathbf{C} P^{2} \# \overline{\mathbf{C} P^{2}}, S\right)$ 为 $\mathbf{C} P^{2} \# \overline{\mathbf{C} P^{2}}$ 中表示 $u$ 的 2- 纽结. 如果 $u$ 不是素类, 容易验证 $H_{1}\left(\mathbf{C} P^{2} \# \overline{\mathbf{C} P^{2}}-S, Z\right) \neq 0$. 我们在此仅考虑由有单 连通补的 2- 纽结, 因而可以假设 $S$ 表示类 $u=(p+1) \xi+p \eta$, 对某 $p \geqslant 0$. 如在第 1 节中那样, 将 $\mathbf{C} P^{2} \# \overline{\mathbf{C} P^{2}}$ 视为 $S^{2}$ 上的非平凡 $S^{2}$ - 丛 $T$. 对每个 $p$, 有 $T$ 到 $S O(2)$ 丛 $B_{2 p+1}$ 的约化. 因而 $\sum_{2 p+1}$ 是表示 $u$ 的光滑嵌人球. 因而 $\left(\mathbf{C} P^{2} \# \overline{\mathbf{C} P^{2}}, \sum_{2 p+1}\right)$ 是表示类 $u=(p+1) \xi+p \eta$ 的 2- 纽结. 注意 $\sum_{2 p+1}$ 的外部微分同胚于 $S^{2}$ 上的 $D^{2}-$ 丛 $D(2 p+1)$, 其 Euler 数为 $2 p+1$.

定理 1 设 $S$ 是 $\mathbf{C} P^{2} \# \overline{\mathbf{C} P^{2}}$ 中的 2- 纽结, 表示类 $u=(p+1) \xi+p \eta \in H_{2}\left(\mathbf{C} P^{2} \# \overline{\left.\mathbf{C} P^{2}, Z\right), p \geqslant 0 .}\right.$ 则 $\pi_{1}\left(\mathbf{C} P^{2} \# \overline{\mathbf{C} P^{2}}-S\right)=0$ 当且仅当有如下的同胚 $f$

$$
f:\left(\mathbf{C} P^{2} \# \overline{\mathbf{C} P^{2}}, S\right) \rightarrow\left(\mathbf{C} P^{2} \# \overline{\mathbf{C} P^{2}}, \sum_{2 p+1}\right) .
$$

设 $S$ 是 $\mathbf{C} P^{2} \# \overline{\mathbf{C} P^{2}}$ 中自相交数为 $m$ 的 2-纽结. $S$ 在 $\mathbf{C} P^{2} \# \overline{\mathbf{C} P^{2}}$ 中的管状邻域微分同胚于 $S^{2}$ 上的 $D^{2}$ - 丛 $D(m)$. 若记 $E$ 为 $S$ 的外部, 则所有以 $E$ 为外部的 2- 纽结可以通过粘合 $D(m)$ 的边 界 $L(m, 1)$ 与 $E$ 的边界 $L(m, 1)$ 得到. 由同痕扩张定理, 这些 2- 纽结的同胚类仅依赖于边界粘 合微分同胚的同痕类.

记 $\mu(L(m, 1))$ 为 $L(m, 1)$ 的微分同痕群 (diffeotopy group).

引理 $1^{[4]} \quad$ 对 $|m| \geqslant 2$,

$$
\mu(L(m, 1))=Z_{2} \text {. }
$$

如果视 $L(m, 1)$ 为 $S^{2}$ 上的 $S^{1}$ - 丛, 则 $\mu$ 由 $L(m, 1)$ 的微分同胚 $f$ 生成, 它在 $S^{1}$ 上诱导的映射 是复共轭. 因此, $\mu(L(m, 1))$ 的一个元素可以扩充为 $\left(D(m), v\left(S^{2}\right)\right)$ 的微分同胚, 其中 $v\left(S^{2}\right)$ 是零 截面.

\section{3 引理及定理的证明}

引理 2 设 $S$ 是 $\mathbf{C} P^{2} \# \overline{\mathbf{C} P^{2}}$ 中有单连通补的 2- 纽结. $S \cdot S \neq 0$. 则 $S$ 在 $\mathbf{C} P^{2} \# \overline{\mathbf{C} P^{2}}$ 中的 外部同胚于 $D(2 p+1)$, 对某非负整数 $p$.

证 不失一般性,我们设 $S$ 表示类 $u=(p+1) \xi+p \eta, p \geqslant 0$. 设 $N(S)$ 是 $S$ 的管状邻域. 则 $N(S)$ 微分同胚于 $D(2 p+1)$. 因而 $E$ 的边界 $\partial E=\partial N(S)$ 是透镜空间 $L(2 p+1,1)$.

容易证明

$$
H_{1}(E, z) \cong H_{3}(E, Z) \cong 0, \quad H_{2}(E, Z)=Z .
$$

考虑以下的交换图.

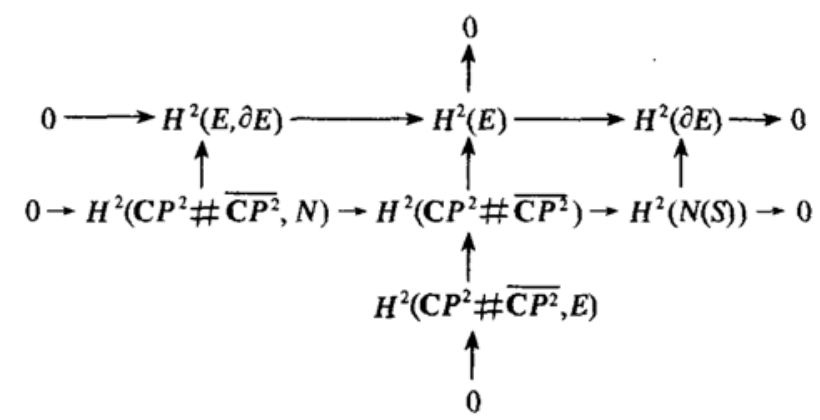

$H^{2}(N(S))$ 由 $u$ 的 Poincaré 对偶生成. $H_{2}(E)$ 中的元素与 $H_{2}(N(S), \partial N(S))$ 中的元相交数为 0 . 
故不难证明 $H^{2}(E, \partial E)$ 由类 $p \xi+(p+1) \eta$ 的对偶生成. 从而 $H_{2}(E, Z)$ 的相交形式 $\left(H_{2}(E, Z),<\right.$, $>)$ 同构.于 $\left(Z,-L_{2 p+1}\right)$, 其中 $-L_{2 p+1}: Z \times Z \rightarrow Z$ 是如下定义的双线性映射 $-L_{2 p+1}(m, n)$ $=-(2 p+1) m n$. 类似于第 1 节中, 将 $\mathbf{C} P^{2} \# \overline{\mathbf{C} P^{2}}$ 看作 $B_{2 p+1}$. 则作为同调类 $u=\sum_{2 p+1}$. 注意 $\sum_{2 p+1}$ 与 $\sigma_{2 p+1}$ 生成 $H_{2}\left(B_{2 p+1}, z\right)$, 所以 $H_{2}(E, \partial E)$ 的一个生成元 $u$ 可以由 $\sigma_{2 p+1}$ 与 $E$ 的交表示. 因 为 $u \cdot \sigma_{2 p+1}=\sum_{2 p+1} \cdot \sigma_{2 p+1}=1$, 故 $\partial u \in H_{1}(L(2 p+1,1), z)$ 可以由 $S^{2}$ 上的 $D^{2}-$ 丛 $N(S)$ 的 $\partial D^{2}$ - 纤 维表示. 注意我们这里研究的是光滑流形, 文献 [5] 中的 (5.4) - (5.6) 节表明 $E$ 同胚于 $D(2 p+1)$.

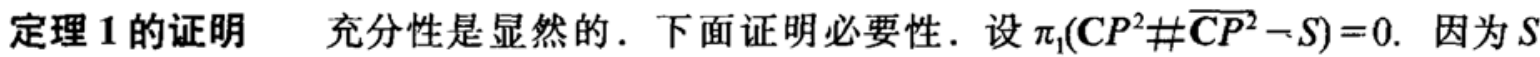
在 $\mathbf{C} P^{2} \# \overline{\mathbf{C} P^{2}}$ 中的管状邻域微分同胚于 $D(2 p+1)$, 我们有 $\left(\mathbf{C} P^{2} \# \overline{\mathbf{C} P^{2}}, S\right) \cong\left(D(2 p+1) \bigcup_{f} E, v\left(S^{2}\right)\right)$, 其中 $f: L(2 p+1,1) \rightarrow L(2 p+1,1)$ 是粘合微分同肧, $v: S^{2} \rightarrow D(2 p+1)$ 是零截面. 由引理 2 , 存在 同胚 $g: E \rightarrow D(2 p+1)$. 记 $\hat{g}=\left.g\right|_{\partial E}$, 则有同胚 $D(2 p+1) \cup_{f} E \cong D(2 p+1) \cup_{\tilde{g} \cdot f} D(2 p+1)$. 由引理 1 , 微分同胚 $g \circ f: L(2 p+1,1) \rightarrow L(2 p+1,1)$ 可扩充为 $\left(D(2 p+1), v\left(S^{2}\right)\right)$ 的微分同胚 $h$, 对 $p \neq 0$. 定义所需要的同胚 $\varphi:\left(D(2 p+1) \bigcup_{f} E, v\left(S^{2}\right)\right) \rightarrow\left(D(2 p+1) \bigcup_{i d} D(2 p+1), v\left(S^{2}\right)\right)$ 为

$$
\varphi= \begin{cases}h, & \text { 在 } D(2 p+1) \text { 上; } \\ g, & \text { 在 } E \text { 上. }\end{cases}
$$

从而我们证明了 $\left(\mathbf{C} P^{2} \# \overline{\mathbf{C} P^{2}}, S\right)$ 同胚于 $\left(\mathbf{C} P^{2} \# \overline{\mathbf{C} P^{2}}, \sum_{2 p+1}\right), p \neq 0$. 对 $p=0, D(1)$ 的边界微分 同胚于 $S^{3}$. 微分同胚 $\tilde{g} \circ f: S^{3} \rightarrow S^{3}$ 或同痕于 $S^{3}$ 的映等映射或同痕于标准的映射度 -1 的映 射. 它将都能扩充为 $\left(D(1), v\left(S^{2}\right)\right)$ 的同胚. 同样的方法可证明定理在 $p=0$ 时亦成立.

Quinn 证明了单连通 4 维流形的同胚群 (homeotopygroup) 同构于其二次型的自同构群 ${ }^{[6 .}$. 利用这个结果, 可以证明以下定理.

定理 2 设 $S$ 是 $\mathbf{C} P^{2} \# \overline{\mathbf{C} P^{2}}$ 中的光滑 2- 纽结, $S \cdot S \neq 0$. 若 $\pi_{1}\left(\mathbf{C} P^{2} \# \overline{\mathbf{C} P^{2}}-S\right)=0$, 则 $S$ 保定向同痕 (ambient isotopic) 千某个 $\sum_{2 p+1}$.

证 Aut $\left(H_{2}\left(\mathbf{C} P^{2} \# \overline{\mathbf{C} P^{2}}, Z\right),<,>\right)$

$$
\begin{aligned}
& =\left\{A \in G L(2, z) \mid A^{2}\left(\begin{array}{cc}
1 & 0 \\
0 & -1
\end{array}\right) A=\left(\begin{array}{cc}
1 & 0 \\
0 & -1
\end{array}\right)\right\} \\
& =\left\{ \pm\left(\begin{array}{ll}
1 & 0 \\
0 & 1
\end{array}\right), \pm\left(\begin{array}{cc}
1 & 0 \\
0 & -1
\end{array}\right)\right\} .
\end{aligned}
$$

对于 $\pi_{1}\left(\mathbf{C} P^{2} \# \overline{\mathbf{C} P^{2}}-S\right)=0$ 及 $S \cdot S \neq 0$, 我们可以假设 $S$ 表示同调类 $(p+1) \xi+p \eta \in H_{2}\left(\mathbf{C} P^{2} \# \overline{\mathbf{C} P^{2}}\right.$, $Z$ ). 由定理 1 , 有同泼

$$
\varphi:\left(\mathbf{C} P^{2} \# \overline{\mathbf{C} P^{2}}, S\right) \rightarrow\left(\mathbf{C} P^{2} \# \overline{\mathbf{C} P^{2}}, \sum_{2 p+1}\right),
$$

它诱导了 $\mathrm{H}_{2}\left(\mathbf{C} P^{2} \# \overline{\mathbf{C} P^{2}}, Z\right)$ 的同构 $\varphi_{*}, \varphi_{*}[S]=\left[\sum_{2 p+1}\right]=(p+1) \xi+p \eta$.

从而

$$
\varphi_{*}= \begin{cases}\left(\begin{array}{ll}
1 & 0 \\
0 & 1
\end{array}\right), & \text { 若 } p \neq 0 ; \\
\left(\begin{array}{ll}
1 & 0 \\
0 & 1
\end{array}\right) & \text { 或 }\left(\begin{array}{cc}
1 & 0 \\
0 & -1
\end{array}\right), \text { 若 } p=0 .\end{cases}
$$

因而对于 $p \neq 0, \varphi$ 同痕于恒等映射. 若 $p=0$, 存在同胚 $\psi:\left(\mathbf{C} P^{2} \# \overline{\mathbf{C} P^{2}, \Sigma_{1}}\right) \rightarrow\left(\mathbf{C} P^{2} \# \overline{\mathbf{C} P^{2}}, \Sigma_{1}\right)$, 
使得 $\psi_{*}=\left(\begin{array}{rr}1 & 0 \\ 0 & -1\end{array}\right)$.

若 $\varphi_{*}=\left(\begin{array}{rr}1 & 0 \\ 0 & -1\end{array}\right)$, 则 $\psi_{*} \cdot \varphi_{*}=\left(\begin{array}{rr}1 & 0 \\ 0 & -1\end{array}\right)$. 因而 $\psi \cdot \varphi$ 是一个同胚 $\left(\mathbf{C} P^{2} \# \overline{\left.\mathbf{C} P^{2}, S\right) \rightarrow}\right.$ $\left(\mathbf{C} P^{2} \# \overline{\mathbf{C}} P^{2}, \Sigma_{1}\right)$.

由定理 2, 立刻有

推论 若 $\xi$ 是 $H_{2}\left(\mathbf{C} P^{2} \# \overline{\mathbf{C} P^{2}}, Z\right)$ 中对应于 $\mathbf{C} P^{2}$ 的生成元, 则任何两个表示 $\xi$ 的光滑嵌人 球同痕.

同样可证明, 若在推论中将 $\mathbf{C} \boldsymbol{P}^{2} \# \overline{\mathbf{C} \boldsymbol{P}^{2}}$ 换成 $\mathbf{C} \boldsymbol{P}^{2}$ 结论仍成立. 这也是四维 Poincaré 猜想 的推论.

致谢感谢李邦河老师的指导和帮助.

\section{参考文献}

[1] Steenrod, N., The Topology of Fibre Bundle, Princeton Univ. Press, 1951.

[2] Wall, C. T. C., Jour. London Math. Soc., 1964, 39:131- 140.

[3] Lawson, T., Michigan Math. Jour., 1987, 34:85-91.

[4] Bonahon, F., Topology, 1983, 22: 305-314.

[5] Boyer, S., Trans. Amer. Math. Soc., 1986, 288: 331- 357.

[6] Quinn, F., Jour. Diff. Geom., 1986, 24:343-372. 